\title{
Bacterial community composition of flocculent matter under a salmonid aquaculture site in Newfoundland, Canada
}

\author{
Joost T. P. Verhoeven ${ }^{1}$, Flora Salvo ${ }^{2}$, Dounia Hamoutene ${ }^{2}$, Suzanne C. Dufour ${ }^{1, *}$ \\ ${ }^{1}$ Department of Biology, Memorial University of Newfoundland, St. John's, NL A1B 3X9, Canada \\ ${ }^{2}$ Science Branch, Fisheries and Oceans Canada, PO Box 5667, St. John's, NL A1C 5X1, Canada
}

\begin{abstract}
Aquaculture has become a rapidly growing industry: over the past 3 decades, commercial production has steadily increased, and further expansion seems likely. However, the rise of aquaculture has been accompanied by concerns, especially regarding environmental sustainability. Substrates located under aquaculture sites receive large influxes of organic matter that can subsequently create anoxic conditions and thereby impact existing benthic communities. Shifts in the relative abundance of specific groups of bacteria could prove to be important indicators of impact and remediation. Here, we investigated bacterial community composition via 16S rRNA gene sequencing on isolated DNA from flocculent matter samples and associated bacterial mats under a hard-bottom aquaculture site in Newfoundland, Canada. We describe the heterogeneous community present in the flocculent matter, characterized by high relative abundances of the genera Spirochaeta (12\%), Prolixibacter (5.6\%) and Marinifilum (4.6\%). Bacterial mats were not composed of Beggiatoa as often hypothesized, but instead were dominated by the genera Spirochaeta $(15 \%)$, Prevotella $(21 \%)$, Meniscus $(11 \%)$ and Odoribacter $(20 \%)$. Our findings provide insights into the bacterial composition of flocculent matter deposited on hard substrates and undergoing degradation, and point to 3 unexpected bacterial genera as potential indicators of organic enrichment.
\end{abstract}

KEY WORDS: Microbiome - Organic enrichment - Bacterial mats - Spirochaeta $\cdot$ Prolixibacter Marinifilum

\section{INTRODUCTION}

In recent years, the aquaculture industry has experienced rapid growth worldwide (Asche et al. 2008), substantiating the hypothesis that aquaculture could soon produce more than half of the seafood consumed globally (Costa-Pierce 2010). Marine aquaculture operations in coastal Newfoundland (NL), Canada, notably the cage culture of salmonids and long-line mussel culture, have seen significant growth in the last decade (Anderson et al. 2005, Fisheries and Oceans Canada 2016).

Along with the increase in finfish aquaculture sites (or 'farms'), concerns regarding ecological impacts

\footnotetext{
${ }^{*}$ Corresponding author: sdufour@mun.ca
}

and sustainability have arisen (Jusup et al. 2009). One major environmental concern is the increased deposition of organic matter in the benthic environment, which usually occurs in the form of a complex mixture known as flocculent matter composed of decomposing fish-food pellets, microbes, fish faeces and other organic matter (Salvo et al. 2015). The accumulation of organic matter on the seafloor can drive an increase in oxygen consumption by environmental or fish faeces-associated micro-organisms (Tett 2008). The resulting hypoxic conditions can negatively, and potentially permanently, affect the benthic in- and epifauna (Karakassis et al. 2000, Jusup et al. 2009, Pochon et al. 2015).

(c) Fisheries and Oceans Canada and J. T. P. Verhoeven, S. C. Dufour 2016. Open Access under Creative Commons by Attribution Licence. Use, distribution and reproduction are unrestricted. Authors and original publication must be credited.

Publisher: Inter-Research · www.int-res.com 
Monitoring the ecological impact of fish farm operations on the environment is key to assess, understand and develop sustainable models for aquaculture. To this end, sediment sulphides and redox potential measurements are often used as indicators to extrapolate the biological response to organic enrichment (Hargrave et al. 2008). In NL, many aquaculture operations occur in bays with water depths over $30 \mathrm{~m}$ and hard substrates comprised of rock and cobble, where grab sampling cannot be conducted, and therefore completing valid sulphide and redox potential measurements is difficult (Hamoutene et al. 2014, 2015).

More recently, visual monitoring has documented the close association of flocculent matter and white Beggiatoa-like bacterial mats with the elevated sulphide levels typically found near farms in NL (Hamoutene 2014). When the collection of sulphides and redox potential measurements is not feasible, drop-camera based observation of flocculent matter and bacterial mats is a valid alternative indicator of organic enrichment, as these features are generally not observed in areas where no active aquaculture operations are conducted (Crawford et al. 2001, Hamoutene 2014, Hamoutene et al. 2015). The formation of bacterial mats in response to aquaculture operations is indicative of a shift in bacterial community composition in the farm vicinity. Indeed, previous studies have shown fish farm-dependent organic enrichment and anoxia to be closely associated with large-scale shifts in bacterial communities (Asami et al. 2005, Dowle et al. 2015). Such shifts are usually characterized by an increase in abundance of sulphur, nitrogen and methane cycle-associated bacteria in the direct environment (Asami et al. 2005, Dowle et al. 2015).

The apparent plasticity of the microbial community in response to aquaculture operations suggests that these communities could have intrinsic indicator potential for indirectly assessing ecological impacts and remediation. However, shifts in microbial community composition are not fully understood, and many questions remain as to what drives these changes. For example, the white bacterial mats observed near farms, until recently considered to be Beggiatoa, have not yet been identified (Dowle et al. 2015). Furthermore, bacterial changes in one region might not be comparable to those observed elsewhere due to differences in geographical location, bathymetry and other environmental factors. In NL, for example, there is often very little natural sediment, and flocculent matter build-up represents a drastic change of substrate for benthic organisms (Hamoutene et al. 2016).
The presence of bacterial mats and flocculent matter are used as indicators in regulatory frameworks for aquaculture (Hamoutene et al. 2014). Further investigating the microbial community composition of flocculent matter and identifying key bacterial species involved is a crucial step in developing our understanding of how aquaculture operations initiate bacterial community changes and whether these shifts can be used to measure impact and, potentially, remediation.

In this study, we investigated the bacterial community composition of multiple flocculent matter samples under a salmonid farm in the Hermitage Bay area, on the south coast of NL. Our analysis was performed by sequencing the V6/V8/V9 region of the 16S rRNA gene. Multiple samples were analysed to detect withinsite variation and determine the homogeneity of the flocculent matter observed. We also collected multiple samples of bacterial mats and attempted to identify the bacterial community driving mat formation.

\section{MATERIALS AND METHODS}

\section{Collection of flocculent matter}

Samples of flocculent matter were collected on 27 August 2015 at a salmonid aquaculture site in 'Little Passage', located between Bay D'Espoir and Hermitage Bay on the south coast of NL. This site, part of a larger ongoing study of benthic remediation processes at aquaculture sites, had been out of production (in fallow) for $3 \mathrm{mo}$ at the time of sampling. Dropcamera visualization was performed to detect stations (locations within the site) presenting flocculent matter and bacterial mats. A total of 7 stations, located approximately $10 \mathrm{~m}$ from each other, were investigated, and an average of 7 grabs (Eckman or box corer), directly adjacent to cage edges, were completed at each station, at an average depth of $72 \mathrm{~m}$. Four stations were selected (based on grab success and retrieved flocculent matter) for molecular analysis, and a total of 15 samples (approximately 4 per station) were collected from grab material using sterile spoons or cut pipette tips. Samples were subsequently stored in sterile containers and frozen at $-20^{\circ} \mathrm{C}$ until arrival at the laboratory, where they were transferred to a $-80^{\circ} \mathrm{C}$ freezer.

\section{DNA extraction and high-throughput sequencing (HTS)}

Flocculent matter samples were thawed on ice and transferred aseptically to bead beating tubes con- 
taining $0.1 \mathrm{~mm}$ glass beads (MO Bio Laboratories). Total microbial DNA was subsequently isolated using the MO BIO PowerViral DNA/RNA isolation kit (MO Bio Laboratories) according to the manufacturer's specifications. Extracted nucleic acids were measured for quality and concentration using a NanoDrop 1000 (Thermo Scientific) and outsourced for Illumina MiSeq 2x300-bp paired-end sequencing of the 16S rRNA (V6/V7/V8 region) gene at the Integrated Microbiome Resource (IMR) at the Centre for Comparative Genomics and Evolutionary Bioinformatics (Dalhousie University, Halifax, Canada), using the previously published primers B969F and B1406R (Comeau et al. 2011).

\section{Processing and taxonomic profiling of HTS data}

Illumina MiSeq sequence data were processed using the in-house developed Streamlined Processor Of Next-gen Sequences (SPONS) pipeline that integrates a multitude of external programs. An initial quality check was performed using Trimmomatic version 0.33 to remove any remaining primer or adapter sequences, check reads for quality (leading and trailing quality threshold $=25$ ) and ensure that the remaining sequence information was of sufficient length (minimum of $100 \mathrm{bp}$ ) (Bolger et al. 2014). Surviving paired-end reads where subsequently merged using PEAR version 0.9 (Zhang et al. 2014). For read merging to occur, a minimal overlap of $105 \mathrm{bp}$ and a $\mathrm{p}$-value of $<0.001$ were required to ensure high-quality sequences. Operational taxonomic units (OTUs) were created through a 2-step process: first the total sequence dataset was de-replicated using VSEARCH (merging strictly identical sequences and adding abundance information), and this de-replicated weighted dataset was successively used in conjunction with the de novo clustering program SWARM to create OTUs (Mahé et al. 2014, Rognes 2016). SWARM was used with adjustment to the default settings to generate fewer OTUs with a higher rate of inclusion (maximum number of differences allowed $=$ 6 , mismatch penalty $=3$, match reward $=6$, gap opening penalty $=10$, gap extension penalty $=3$ ), and the seed of each OTU was kept as a representative sequence. Representative OTU sequences were checked for the existence of chimeras through VSEARCH (UCHIME de novo implementation) using the SILVA SSU reference database release 123 (clustered at $99 \%$ identity). OTU distribution amongst samples was analysed by mapping original reads to the representative OTU sequences using VSEARCH (utilizing USEARCH global implementation, percentage identity $=97 \%$ ).

Taxonomy was inferred for each OTU through an internal SPONS wrapper function which invokes 4 taxonomic classifiers through the assign_taxonomy.py command within the QIIME framework: UCLUST (maximum number of hits to consider $=1$, minimum percent similarity $=0.8$, minimum consensus fraction $=$ 0.76), Ribosomal Database Project (RDP) Classifier (minimum confidence $=0.5$ ), SortMeRNA (minimum consensus fraction $=0.51$, similarity $=0.8$, best alignments per read $=1$, coverage $=0.8$, e-value $=1.0$ ) and BLAST (e-value $=0.001$ ) (Altschul et al. 1990, Wang et al. 2007, Caporaso et al. 2010, Edgar 2010, Kopylova et al. 2012). After completion, a consensus taxonomy was extracted for each OTU at the lowest common denominator where at least 3 classifier methods were in agreement. All classification methods used the fully redundant SILVA SSU reference database and taxonomy (release 123) (Quast et al. 2013, Yilmaz et al. 2014). As an additional filtering step, OTUs with taxonomical assignments other than Bacteria where removed from the dataset. Full dataset phylogeny was created by generating alignments of OTU sequences in MAFFT7 using the FFT-NS-2 algorithm which were subsequently trimmed using gBlocks version $0.91 \mathrm{~b}$ (minimum block length $=3$, all gap positions allowed) and used for construction of an approximately-maximum-likelihood phylogenetic tree by FastTree version 2.1.8 (fastest option enabled in combination with the generalised time reversible model) (Castresana 2000, Price et al. 2010, Katoh \& Standley 2013). The source code of the SPONS pipeline is available upon request.

\section{Bacterial diversity analysis, visualization and statistics}

Alpha diversity was investigated using QIIMEs alpha_rarefaction.py workflow using both Shannon's diversity index (shannon) and Simpson's evenness measure E (simpson_e) (Faith \& Baker 2007). Sample OTU data were converted to relative abundance by dividing counts by total library size using QIIMEs summarize_taxa.py. Subsequently, heatmap analysis was performed in $\mathrm{R}$ using additional features from the R package PhyloSeq (McMurdie \& Holmes 2013, R Core Team 2015). Supporting dendrograms for heatmaps were created using hierarchical clustering (agglomeration method: 'average') of Bray-Curtis dissimilarity generated from relative abundance transformed OTU count data, at phylum and genus levels, with the community ecology R package 'vegan' (Oksanen et al. 2016). Relative abundance data were 
imported into PRIMER 6 with the PERMANOVA+ add-on for further analysis (Clarke 1993, Clarke \& Gorley 2006, Anderson et al. 2008). Resemblance matrices were created by analysing differences between samples using the Bray-Curtis dissimilarity measure. Following the construction of the similarity matrix, a hierarchical cluster analysis was performed with the group average cluster mode. Principal coordinate analysis (PCO) was conducted on the similarity matrix, constrained to a maximum of 14 principal coordinates. Hierarchical cluster analysis data were used to overlay clusters at a resemblance level of $40 \%$, while simultaneously plotting vectors using the Pearson correlation type. Displayed vectors were manually set to match high abundance taxa. As relative abundance based analysis can be prone to distortion by excessively abundant or rare taxa, results were validated by repeating the analysis workflow (data not shown) using OTU count data normalized with the variance stabilization transformation as implemented in the R package DESeq2 (Love et al. 2014). This method was used to remove the dependence of the variance on the mean and produced $\log _{2}-$ transformed data, normalized with respect to library size (Love et al. 2014).

\section{RESULTS}

\section{Description of samples}

Flocculent matter showed significant visual heterogeneity within and among grab samples. Flocculent matter was either black (Fig. 1A) or brown (Fig. 1B). No intact bacterial mats as seen on videos from stations were recovered; however, small remnants of presumed bacterial mats were observed and sampled (Fig. 1C). One grab retrieved visually and physically distinct thick, light brown material which upon surfacing expanded to over 3 times its original volume while forming bubbles and venting a gaseous substance with a sulphurous odour (Fig. 1D, further referred to as 'gaseous'). From the grabs, we collected a total of 15 samples, of which 10 consisted of bulk flocculent matter, 4 were more finely sampled bacterial mats, and 1 was collected from the aforementioned gaseous flocculent matter.

\section{Inspection of HTS data}

HTS of the V6/V7/V8 16S rRNA gene region yielded over 383000 merged read-pairs passing quality
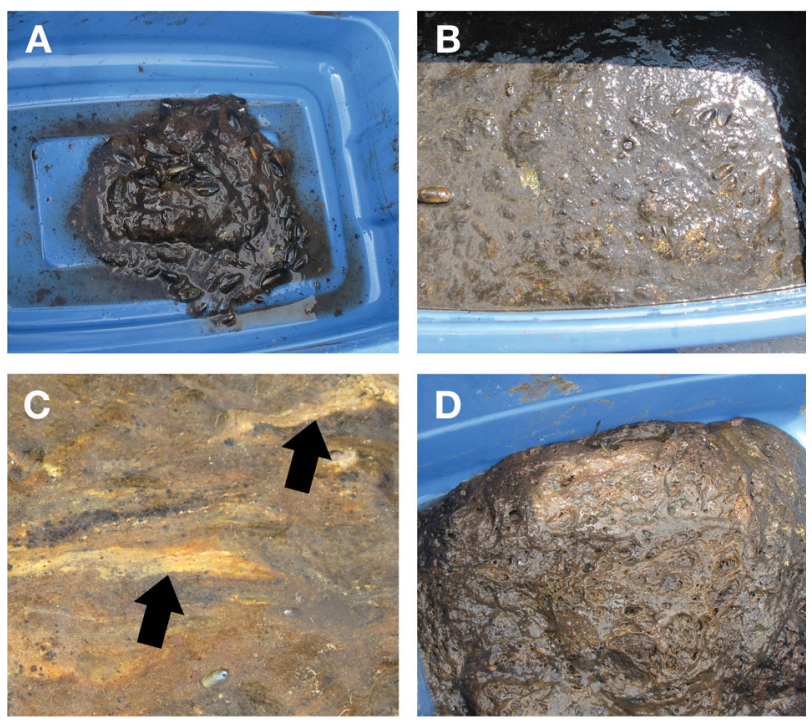

Fig. 1. Flocculent matter retrieved by grab sampling under a hard-bottom aquaculture site in Newfoundland, Canada. Visually distinct material is shown, including (A) dark/black and (B) brown flocculent matter, (C) suspected bacterial mat remains (indicated by arrowhead) on top of flocculent matter and (D) 'gaseous' flocculent matter

checks, with an average read depth of 18186 sequences sample ${ }^{-1}$. Downstream of quality checking, chimeric removal and clustering of sequences, 4459 high-quality, non-singleton microbial OTUs were detected amongst samples. Rarefaction curve analysis of OTU data indicated that all samples reached their diversity plateau, indicating sufficient sequencing depth (Fig. A1 in the Appendix). Sequence data were deposited in the NCBI short read archive (SRA) linked to BioProject accession number PRJNA352252.

\section{Bacterial community diversity and structure}

Bulk flocculent matter samples $(\mathrm{n}=10)$ showed a significantly $(\mathrm{p}<0.001)$ higher average bacterial diversity $\left(H^{\prime}=7.8590\right)$ than bacterial mat $(\mathrm{n}=4)$ samples $\left(H^{\prime}=6.1280\right)$. A similar trend was observed for community evenness, with bulk samples presenting a significantly higher $(\mathrm{p}=0.05)$ evenness $(E=0.066)$ when compared to mat samples $(E=0.0154)$.

Analysis of the most abundant taxa (Fig. 2A) indicated that the bacterial community associated with the flocculent matter in this study was mainly dominated by the classes Bacteroidia, Spirochaetes, Clostridia and Cytophagia. Interestingly, 3 distinct clusters were observed, showing a fluctuation in the abundance of the Bacteroidia and Epsilonproteobacteria. 
A

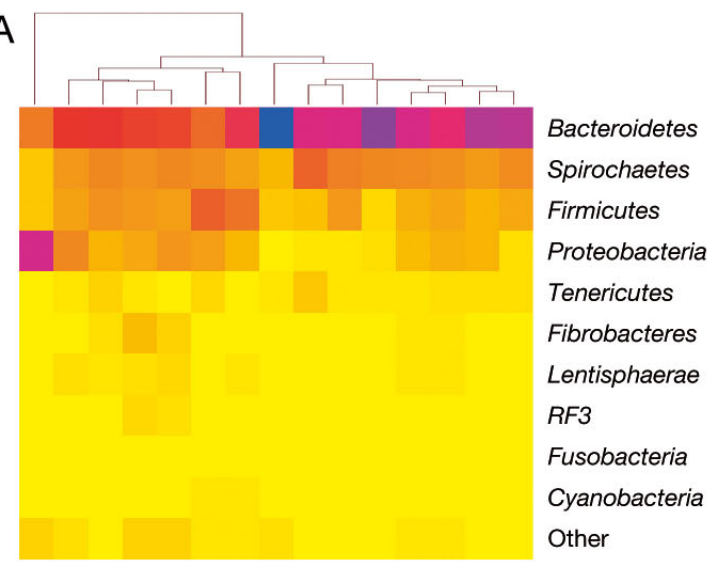

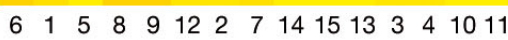

Sequence abundance (\%)

Fig. 2. Heat map visualization of the abundance and distribution of the 10 most abundant taxonomical groups found in aquaculture site flocculent matter. Indicated in rows are the most abundant (A) phyla and (B) genera. Colours indicate relative sequence abundance $(\%)$, columns represent individual samples labeled by sample number. A dendrogram constructed from relative abundance transformed operational taxonomic unit (OTU) count data using hierarchical clustering of Bray-Curtis dissimilarity is displayed at the top of both panels

When investigating the abundance data at the genus level (Fig. 2B), the clustering of samples remained, and a clear correlation between clusters and sample type (bulk flocculent matter or bacterial mat) was observed. Mat samples exhibited a high relative abundance of a small group of 4 taxa, comprising the majority of the detected bacterial population, in line with the observed $H^{\prime}$ and $E$ values. This was in contrast to the bulk flocculent matter samples, which displayed a more even distribution of bacterial taxa, with less dominance.

The mat-specific bacterial community was dominated by the genera Odoribacter $(20 \%)$, Spirochaeta $(15 \%)$, Prevotella $(21 \%)$ and Meniscus (11\%). The gaseous sample (sample 7) also showed a high abundance of Odoribacter (58\%) and Meniscus (18\%) and appeared similar in composition to the mat-specific community. In contrast, bulk flocculent matter samples were not dominated by Odoribacter, which had a relative abundance of only $1.9 \%$. Similarly, the genera Prevotella and Meniscus also showed a sharp decrease in the bulk flocculent matter sample group, with relative abundances of 7 and $5 \%$, respectively. The Spirochaeta only displayed a small difference in abundance $(12 \%)$ in the bulk flocculent samples compared to the bacterial mat samples. Sample 6, a black flocculent matter sample, displayed a highly divergent bacterial community with an extremely high abundance of Epsilonproteobacteria, driven by the genus
B

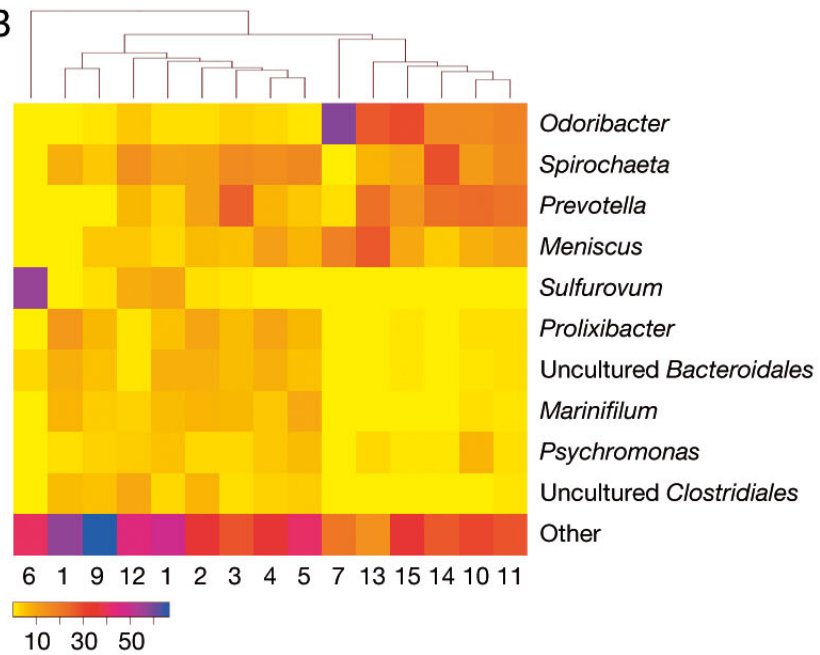

$1030 \quad 50$

Sequence abundance (\%)
Sulfurovum comprising more than half of the bacterial community in this sample (56\%).

Similar results were obtained by investigating samples using hierarchical clustering based on Bray-Curtis dissimilarity between samples (Fig. 3). Again, at $40 \%$ similarity, 3 distinct clusters were observed: (1) the highly dissimilar sample 6 ; $(2)$ a cluster of 8 samples (bulk flocculent matter type only); and (3) a cluster of 6 samples including all of the bacterial mat samples, 1 bulk flocculent matter sample and the gaseous sample as an outgroup.

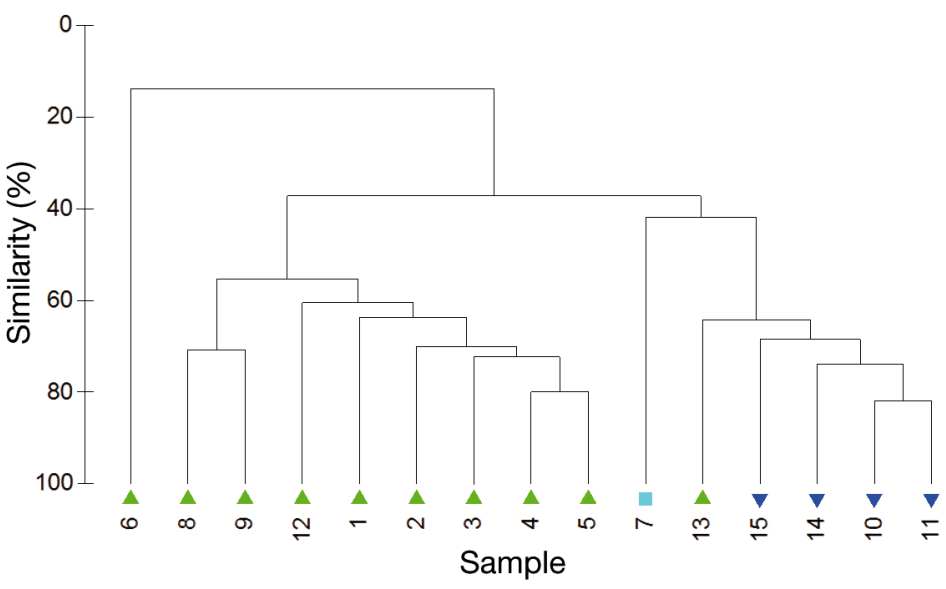

Fig. 3. Cluster analysis of flocculent matter samples. Dendrogram shows the mat-specific and bulk flocculent matter samples as separate clusters. Dendrogram is constructed using hierarchical clustering (using group-average linking) based on Bray-Curtis dissimilarity measurements. Symbols indicate sample type; green triangles: bulk flocculent matter, blue triangles: bacterial mat samples, square: gaseous sample 


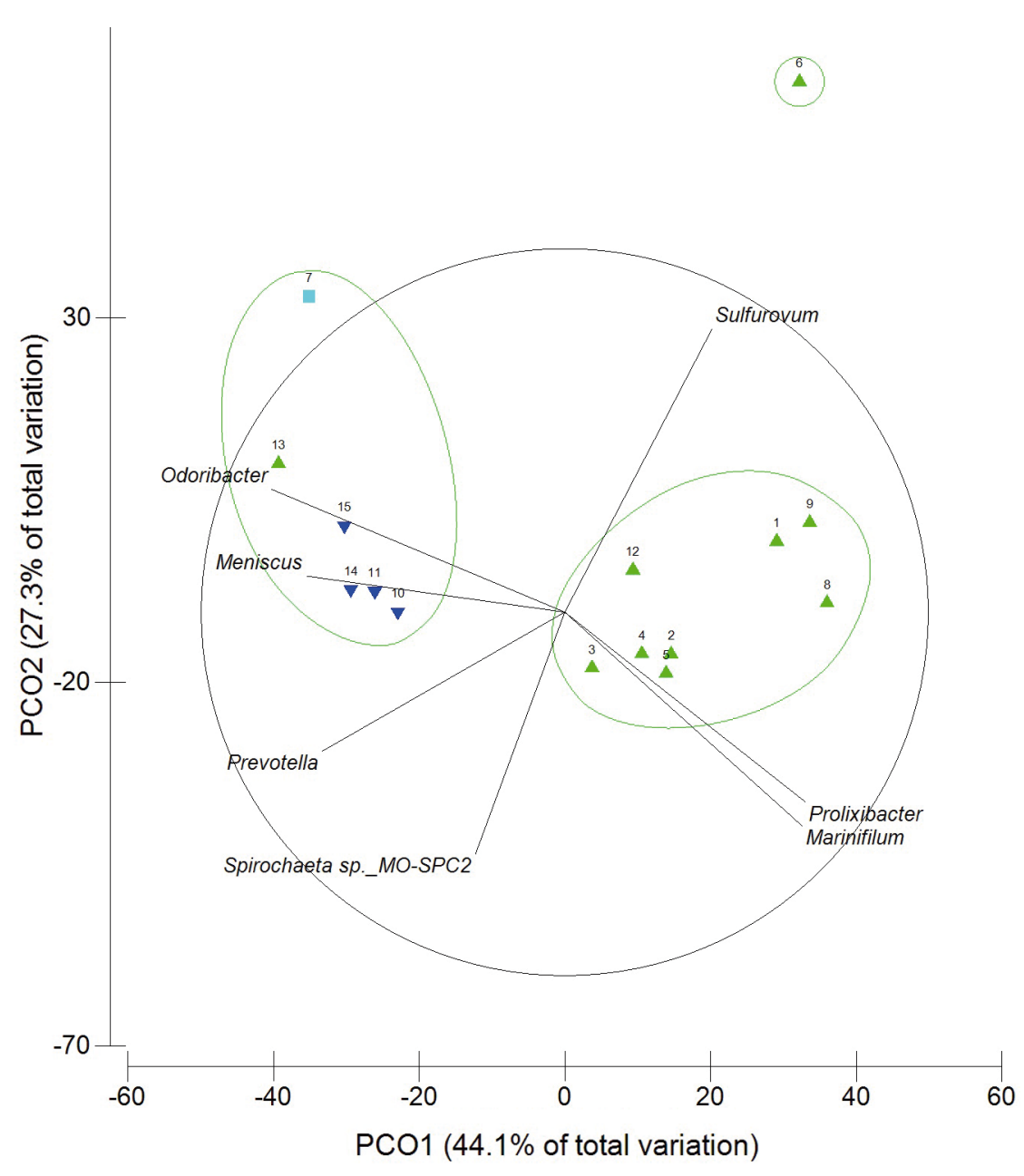

Fig. 4. Principal coordinate (PCO) analysis of flocculent matter samples. Indicated are sample clusters by $40 \%$ similarity (green line). Symbols and numbers indicate sample and sample type; green triangles: bulk flocculent matter, blue triangles: bacterial mat samples, square: gaseous sample. PCO vectors

(Pearson correlation, solid lines) of high abundance genera are shown

\section{DISCUSSION}

As the aquaculture industry continues to expand, understanding the impacts of such operations on the natural environments in which they are performed becomes essential. Bacterial communities are highly sensitive to changes in the environment and have therefore been proposed as indicators of ecological impacts of aquaculture and its remediation over time (Asami et al. 2005, Hamoutene et al. 2014, Dowle et al. 2015). We investigated the bacterial composition of flocculent matter present under an aquaculture site with hard-bottom substrate in NL. A total of 15 flocculent matter samples, heterogeneous in physical appearance, were retrieved and originally categorized as 4 different sample types: brown, black and gaseous matter, as well as bacterial mats (Fig. 1).

\section{Bulk of the flocculent matter}

Similarity analysis on the community structure revealed that brown and black flocculent matter showed only relatively small differences amongst samples (Fig. 4), indicating that the bulk of the flocculent matter harbours a comparatively stable bacterial community. These sample types were dominated by the classes Bacteroidia, Spirochaetes and Clostridia. At the

PCO analysis (Fig. 4) revealed the cluster of bacterial mat and gaseous samples to be clearly distinct from the cluster of bulk flocculent matter samples along the first principal coordinate (PCO1), accounting for $44.1 \%$ of the total variation observed in the dataset. The second principal coordinate (PCO2) accounted for $27.3 \%$ of the total variation, where sample 7 (gaseous) and sample 6 (Sulfurovum overabundant), through the higher abundance of Odoribacter and Sulfurovum, respectively, were the main drivers for variation along this coordinate. Vectors in the PCO analysis identified similar genera as obtained from the abundance data analysis as being correlated with clusters, further highlighting the involvement of Odoribacter, Meniscus and Prevotella in distinguishing the mat sample group, and the affinity of Prolixibacter and Marinifilum with the bulk flocculent matter samples. genus level, the community composition was diffuse, and no clearly dominant taxa were observed. However, PCO analysis results showed the genera Spirochaeta, Prolixibacter and Marinifilum to be the unique drivers for this cluster.

The Spirochaeta are ubiquitous inhabitants of many aquatic environments including lakes, oceans and marine sediments (Holt 1994). As they are commonly found in marine environments and metabolise carbohydrates such as those found in flocculent matter, we hypothesized that the presence of Spirochaeta is most likely the result of colonization of these bacteria from the water column into the favourable conditions of flocculent matter. Less commonly observed in the environment are the Prolixibacter. Currently there are 2 species described for Prolixibacter, both fermenting carbohydrates (Holmes et al. 2007, lino et al. 2015). The more recently described $P$. denitrificans is further 
known to reduce nitrate (Iino et al. 2015). It is not known whether these bacteria stem from fish faeces, food pellets or the natural environment. As the ecological role of this genus is currently not well known, it is difficult to predict the functional involvement of Prolixibacter; however, their fermentative and psychrotolerant properties are most likely vital to their success and presence within the flocculent matter. Further associated with the bulk flocculent matter is Marinifilum, another genus comprising facultative anaerobes which ferment a variety of sugars ( $\mathrm{Na}$ et al. 2009, Bozo-Hurtado et al. 2013, Ruvira et al. 2013); similarly to Prolixibacter, Marinifilum are still not well characterized in terms of ecological characteristics. A previous study has shown the Marinifilum to be members of a sulphate-reducing consortium in anoxic waters, potentially indicating a relevant role in sulphur transformation, as well as coupling of carbon and sulphur cycles (Bozo-Hurtado et al. 2013). Notably, the Marinifilum found in this study showed a 95\% sequence identity to the bacterial endosymbiont of Osedax mucofloris (data not shown), a worm previously found to form opportunistic polychaete complexes (OPCs) degrading whale bones (Verna et al. 2010). Similar OPCs have been described under impacted aquaculture sites (Hamoutene et al. 2013, Salvo et al. 2014), raising the question of whether the Marinifilum identified here could represent an endosymbiont of aquaculture-related OPC worms.

Sample 6 showed a highly divergent bacterial composition in which the genus Sulfurovum comprised over half of the microbiome and was the only abundant taxon observed. The sole known species is $S$. lithotrophicum, a strict chemolithoautotrophic sulphur-oxidizing bacterium. Gross morphology of this sample did not differ significantly from other samples, and it is unknown why a pronounced shift occurred in this sample. It does, however, demonstrate the heterogeneous nature of flocculent matter and the existence of highly variable niche micro-environments.

\section{Bacterial mats}

The 4 samples collected as bacterial mats formed a separate cluster that also included 1 bulk flocculent sample and the gaseous sample in a separate, more distant branch (Fig. 3). This cluster revealed a divergent bacterial community compared to the bulk flocculent matter, and enrichment for the Bacteroidia was observed. At the genus level, a distinct higher abundance of Odoribacter, Meniscus and Prevotella was noted (Fig. 2). Accompanying this dominance was the lower presence of other bacterial taxa in these mat samples, further supported by the low diversity and evenness values (Table 1). Bacterial mats all displayed a low diversity, low evenness index and the enrichment of specific bacterial members. Based on abundance data and PCO analysis (Fig. 4), we hypothesize that the genera Prevotella, Meniscus and Odoribacter could be key players in bacterial mat establishment and persistence as observed near aquaculture sites. This is divergent from previous studies that proposed a role for Beggiatoa in the bacterial mat-forming process. The absence of Beggiatoa as an abundant or enriched genus echoes the recent report of only low levels of Beggiatoa in sediments beneath aquaculture sites, with other bacteria thought to be involved in mat formation (Dowle et al. 2015).

The Prevotella have been isolated from a number of niches including the human oral cavity, skin abscesses and terrestrial soils, as members of the anaerobic, hydrogen sulphide $\left(\mathrm{H}_{2} \mathrm{~S}\right)$-producing bacterial community (Washio et al. 2005, Purushe et al. 2010). There is evidence that members of the genus Prevotella are capable of metabolising various poly- 
saccharides, simple sugars and pectin, in combination with the scavenging of peptides and ammonia as nitrogen sources (Purushe et al. 2010), all substrates which are expected to be present in flocculent matter. Species within the anaerobic genus Odoribacter display similar characteristics and can metabolise various carbohydrates and nitrogenous substrates, et al. 2011). Interestingly, abundance and PCO analysis data showed that this genus almost completely replaced all other taxa within the gaseous sample and drove the divergence of this sample. It is likely that Odoribacter generated the highly expanding gaseous substance sampled in this study. Lastly, the genus Meniscus, containing the sole species M. glaucopis, is associated with high organic enrichment such as in wastewater treatment plants and industrial effluents, but can also be present in the hypolimnion of lakes and marine waters (Irgens 1977, Krieg et al. 2010). When grown to stationary phase in vitro, these aerotolerant anaerobic bacteria migrate to the surface of the culture medium and form a white band. They furthermore display gas vacuoles distributed within cells and potentially exhibit filamentous growth (Irgens et al. 1989). These characteristics of white band formation, gas vacuoles and filamentous growth are reminiscent of those observed in Beggiatoa. Due to visual similarities to Beggiatoa and their increased abundance in bacterial mat samples, $\mathrm{Me}$ niscus represents a novel bacterial candidate which could play a key role in the formation of bacterial mats as observed near aquaculture sites.

\section{Implications of findings}

Our results differ substantially from observations at aquaculture sites in other localities (Asami et al. 2005, Dowle et al. 2015). These previous studies reported high abundances of Desulfobulbaceae (Asami et al. 2005) and Desulfobacterales, Flavobacteriales and Clostridiales (Dowle et al. 2015). While these groups were also observed in the present study, they occurred in low abundance. Conversely, the Bacteroidia, Spirochaetales and Clostridia were not previously detected at the high abundances observed herein. Consideration needs to be given to substrate type, as our study site has minimal natural sediment and therefore differs from sites in other studies. The absence of natural sediment containing a previously established bacterial community, and the resulting deposition of flocculent matter on hard substrates could explain the vast differences in bacterial communities.
Based on our data, we hypothesize that a phased process occurs when flocculent matter is deposited on hard-bottom substrate. Upon flocculent deposition, the active growth of flocculent-associated bacteria as well as environmental bacteria occurs. The metabolic processes and gradual development of an anoxic environment could favour the growth of a small, distinct community as observed in the bacterial mat, where the aerotolerant Meniscus developed at the interface between the water column and flocculent matter. The gaseous sample collected most likely represents an advanced stage of the decomposition process, in which Odoribacter and Meniscus are even further dominating the bacterial community. Additional sample collection, at multiple time points and sites, is needed to confirm this hypothesis. Furthermore, investigating bacterial expression levels could provide significant insights into active biochemical pathways to further understand the decomposition process.

We present a novel description of bacterial communities associated with flocculent matter degradation on hard-bottom substrates. Specific taxa represent important biological indicators of organic enrichment stemming from fish farms. Differences compared to previously published studies, and variation between samples within our study site highlight the need for a more in-depth characterization of bacterial communities under aquaculture sites in order to fully understand microbial responses.

Acknowledgements. We thank Bob Sweeney and affiliated staff from SIMCORP for their invaluable help during field work and sample collection. This work was supported by the Fisheries and Oceans Canada Program for Aquaculture Regulatory Research (PARR) (Grant number: PARR-2015NL-07).

\section{LITERATURE CITED}

Altschul SF, Gish W, Miller W, Myers EW, Lipman DJ (1990) Basic local alignment search tool. J Mol Biol 215:403-410 Anderson MR, Tlusty MF, Pepper VA (2005) Organic enrichment at cold water aquaculture sites - the case of coastal Newfoundland. In: Hargrave BT (ed) Environmental effects of marine finfish aquaculture. Springer, Berlin, p 99-113

Anderson M, Gorley RN, Clarke RK (2008) PERMANOVA+ for Primer: guide to software and statistical methods. PRIMER-E, Plymouth

Asami H, Aida M, Watanabe K (2005) Accelerated sulfur cycle in coastal marine sediment beneath areas of intensive shellfish aquaculture. Appl Environ Microbiol 71: 2925-2933

Asche F, Roll KH, Tveterås S (2008) Future trends in aquaculture: productivity growth and increased production. 
In: Holmer M, Black K, Duarte CM, Marbà N, Karakassis I (eds) Aquaculture in the ecosystem. Springer, Dordrecht, p 271-292

Bolger AM, Lohse M, Usadel B (2014) Trimmomatic: a flexible trimmer for Illumina sequence data. Bioinformatics 30:2114-2120

Bozo-Hurtado L, García-Amado MA, Chistoserdov A, Varela R, Narvaez JJ, Colwell R, Suárez P (2013) Identification of bacteria in enrichment cultures of sulfate reducers in the Cariaco Basin water column employing denaturing gradient gel electrophoresis of $16 \mathrm{~S}$ ribosomal RNA gene fragments. Aquat Biosyst 9:17

> Caporaso JG, Kuczynski J, Stombaugh J, Bittinger K and others (2010) QIIME allows analysis of high-throughput community sequencing data. Nat Methods 7:335-336

Castresana J (2000) Selection of conserved blocks from multiple alignments for their use in phylogenetic analysis. Mol Biol Evol 17:540-552

> Clarke KR (1993) Non-parametric multivariate analyses of changes in community structure. Aust J Ecol 18:117-143

Clarke KR, Gorley RN (2006) PRIMER v6: user manual/ tutorial. PRIMER-E, Plymouth

> Comeau AM, Li WKW, Tremblay JÉ, Carmack EC, Lovejoy C (2011) Arctic Ocean microbial community structure before and after the 2007 record sea ice minimum. PLOS ONE 6:e27492

> Costa-Pierce BA (2010) Sustainable ecological aquaculture systems: the need for a new social contract for aquaculture development. Mar Technol Soc J 44:88-112

Crawford CM, Mitchell IM, Macleod CKA (2001) Video assessment of environmental impacts of salmon farms. ICES J Mar Sci 58:445-452

$>$ Dowle E, Pochon X, Keeley N, Wood SA (2015) Assessing the effects of salmon farming seabed enrichment using bacterial community diversity and high-throughput sequencing. FEMS Microbiol Ecol 91:fiv089

Edgar RC (2010) Search and clustering orders of magnitude faster than BLAST. Bioinformatics 26:2460-2461

Faith DP, Baker AM (2007) Phylogenetic diversity (PD) and biodiversity conservation: some bioinformatics challenges. Evol Bioinform Online 2:121-128

Fisheries and Oceans Canada (2016) Statistics on Aquaculture production quantities and values, 2000-2014. www.dfo-mpo.gc.ca/stats/aqua/aqua-prod-eng.htm (accessed 10 May 2016)

Göker M, Gronow S, Zeytun A, Nolan M and others (2011) Complete genome sequence of Odoribacter splanchnicus type strain (1651/6T). Stand Genomic Sci 4:200-209

Hamoutene D (2014) Sediment sulphides and redox potential associated with spatial coverage of Beggiatoa spp. at finfish aquaculture sites in Newfoundland, Canada. ICES J Mar Sci 71:1153-1157

Hamoutene D, Mabrouk G, Sheppard L, MacSween C, Coughlan E, Grant C (2013) Validating the use of Beggiatoa sp. and opportunistic polychaete worm complex (OPC) as indicators of benthic habitat condition at finfish aquaculture sites in Newfoundland. Can Tech Rep Fish Aquat Sci 3028

Hamoutene D, Sheppard L, Mersereau J, Oldford V and others (2014) Applicability of the use of visual indicators [presence of Beggiatoa and/or Opportunistic Polychaete Complexes (OPC)] to identify benthic changes due to aquaculture on various substrates. Res Doc 2014/063. Canadian Science Advisory Secretariat, Fisheries and Oceans Canada, Ottawa
Hamoutene D, Salvo F, Bungay T, Mabrouk G, Couturier C, Ratsimandresy A, Dufour SC (2015) Assessment of finfish aquaculture effect on Newfoundland epibenthic communities through video monitoring. North Am J Aquacult $77: 117-127$

Hamoutene D, Salvo F, Donnet S, Dufour SC (2016) The usage of visual indicators in regulatory monitoring at hard-bottom finfish aquaculture sites in Newfoundland (Canada). Mar Pollut Bull 108:232-241

Hargrave BT, Holmer M, Newcombe CP (2008) Towards a classification of organic enrichment in marine sediments based on biogeochemical indicators. Mar Pollut Bull 56: $810-824$

Holmes DE, Nevin KP, Woodard TL, Peacock AD, Lovley DR (2007) Prolixibacter bellariivorans gen. nov., sp. nov., a sugar-fermenting, psychrotolerant anaerobe of the phylum Bacteroidetes, isolated from a marine-sediment fuel cell. Int J Syst Evol Microbiol 57:701-707

Holt JG (1994) Bergey's manual of determinative bacteriology. Lippincott Williams \& Wilkins, Philadelphia, PA

Iino T, Sakamoto M, Ohkuma M (2015) Prolixibacter denitrificans sp. nov., an iron-corroding, facultatively aerobic, nitrate-reducing bacterium isolated from crude oil, and emended descriptions of the genus Prolixibacter and Prolixibacter bellariivorans. Int J Syst Evol Microbiol 65: 2865-2869

Irgens RL (1977) Meniscus, a new genus of aerotolerant, gas-vacuolated bacteria. Int J Syst Evol Microbiol 27: $38-43$

Irgens DRL, Suzuki I, Staley JT (1989) Gas vacuolate bacteria obtained from marine waters of Antarctica. Curr Microbiol 18:261-265

Jusup M, Klanjš ek J, Petricioli D, Legovi T (2009) Predicting aquaculture-derived benthic organic enrichment: model validation. Ecol Model 220:2407-2414

Karakassis I, Tsapakis M, Hatziyanni E, Papadopoulou KN, Plaiti W (2000) Impact of cage farming of fish on the seabed in three Mediterranean coastal areas. ICES J Mar Sci 57:1462-1471

> Katoh K, Standley DM (2013) MAFFT multiple sequence alignment software version 7 : improvements in performance and usability. Mol Biol Evol 30:772-780

- Kopylova E, Noé L, Touzet H (2012) SortMeRNA: fast and accurate filtering of ribosomal RNAs in metatranscriptomic data. Bioinformatics 28:3211-3217

Krieg NR, Parte A, Ludwig W, Whitman WB and others (2010) Bergey's manual of systematic bacteriology, Vol 4: the Bacteroidetes, Spirochaetes, Tenericutes (Mollicutes), Acidobacteria, Fibrobacteres, Fusobacteria, Dictyoglomi, Gemmatimonadetes, Lentisphaerae, Verrucomicrobia, Chlamydiae, and Planctomycetes. Springer, New York, NY

Love MI, Huber W, Anders S (2014) Moderated estimation of fold change and dispersion for RNA-seq data with DESeq2. Genome Biol 15:550

Mahé F, Rognes T, Quince C, de Vargas C, Dunthorn M (2014) Swarm: robust and fast clustering method for amplicon-based studies. PeerJ 2:e593

McMurdie PJ, Holmes S (2013) phyloseq: an R package for reproducible interactive analysis and graphics of microbiome census data. PLOS ONE 8:e61217

Na H, Kim S, Moon EY, Chun J (2009) Marinifilum fragile gen. nov., sp. nov., isolated from tidal flat sediment. Int J Syst Evol Microbiol 59:2241-2246

Oksanen J, Blanchet FG, Kindt R, Legendre P and others 
(2016) vegan: community ecology package. http:// CRAN.R-project.org/package=vegan (accessed 18 Oct 2016)

Pochon X, Wood SA, Keeley NB, Lejzerowicz F, Esling P, Drew J, Pawlowski J (2015) Accurate assessment of the impact of salmon farming on benthic sediment enrichment using foraminiferal metabarcoding. Mar Pollut Bull 100:370-382

Price MN, Dehal PS, Arkin AP (2010) FastTree 2 - approximately maximum-likelihood trees for large alignments. PLOS ONE 5:e9490

Purushe J, Fouts DE, Morrison M, White BA and others (2010) Comparative genome analysis of Prevotella ruminicola and Prevotella bryantii: insights into their environmental niche. Microb Ecol 60:721-729

Quast C, Pruesse E, Yilmaz P, Gerken J and others (2013) The SILVA ribosomal RNA gene database project: improved data processing and web-based tools. Nucleic Acids Res 41:D590-D596

R Core Team (2015) R: a language and environment for statistical computing. www.r-project.org

Rognes T (2016) VSEARCH: versatile open-source tool for metagenomics. https://github.com/torognes/vsearch

Ruvira MA, Lucena T, Pujalte MJ, Arahal DR, Macián MC (2013) Marinifilum flexuosum sp. nov., a new Bacteroidetes isolated from coastal Mediterranean Sea water and emended description of the genus Marinifilum Na et al. 2009. Syst Appl Microbiol 36:155-159

Salvo F, Wiklund H, Dufour SC, Hamoutene D, Pohle G, Worsaae K (2014) A new annelid species from whale- bones in Greenland and aquaculture sites in Newfoundland: Ophryotrocha cyclops, sp. nov. (Eunicida: Dorvilleidae). Zootaxa 3887:555-568

Salvo F, Hamoutene D, Dufour SC (2015) Trophic analyses of opportunistic polychaetes (Ophryotrocha cyclops) at salmonid aquaculture sites. J Mar Biol Assoc UK 95: 713-722

Tett P (2008) Fish farm wastes in the ecosystem. In: Holmer M, Black K, Duarte CM, Marbà N, Karakassis I (eds) Aquaculture in the ecosystem. Springer, Dordrecht, p 1-46

Verna C, Ramette A, Wiklund H, Dahlgren TG, Glover AG, Gaill F, Dubilier N (2010) High symbiont diversity in the bone-eating worm Osedax mucofloris from shallow whale-falls in the North Atlantic. Environ Microbiol 12: 2355-2370

> Wang Q, Garrity GM, Tiedje JM, Cole JR (2007) Naïve Bayesian classifier for rapid assignment of rRNA sequences into the new bacterial taxonomy. Appl Environ Microbiol 73:5261-5267

Washio J, Sato T, Koseki T, Takahashi N (2005) Hydrogen sulfide-producing bacteria in tongue biofilm and their relationship with oral malodour. J Med Microbiol 54: 889-895

- Yilmaz P, Parfrey LW, Yarza P, Gerken J and others (2014) The SILVA and 'All-species Living Tree Project (LTP)' taxonomic frameworks. Nucleic Acids Res 42:D643-D648

Z Zhang J, Kobert K, Flouri T, Stamatakis A (2014) PEAR: a fast and accurate Illumina Paired-End reAd mergeR. Bioinformatics 30:614-620

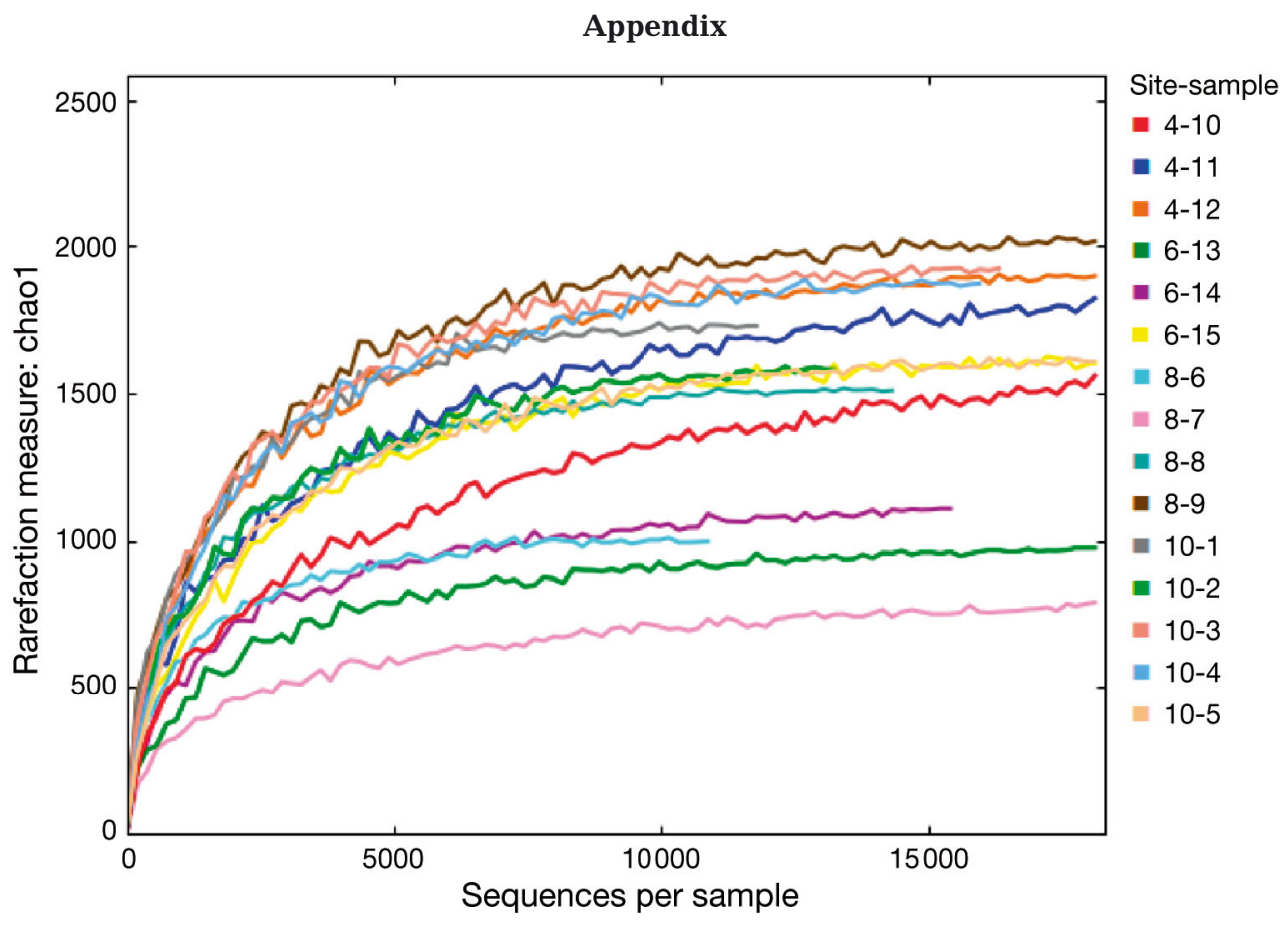

Fig. A1. Rarefaction curves of samples. Shown are individual samples along with the corresponding fraction sampled and calculated Chao1 rarefaction measure.

Editorial responsibility: Marianne Holmer, Odense, Denmark
Submitted: June 2, 2016; Accepted: September 29, 2016 Proofs received from author(s): November 4, 2016 